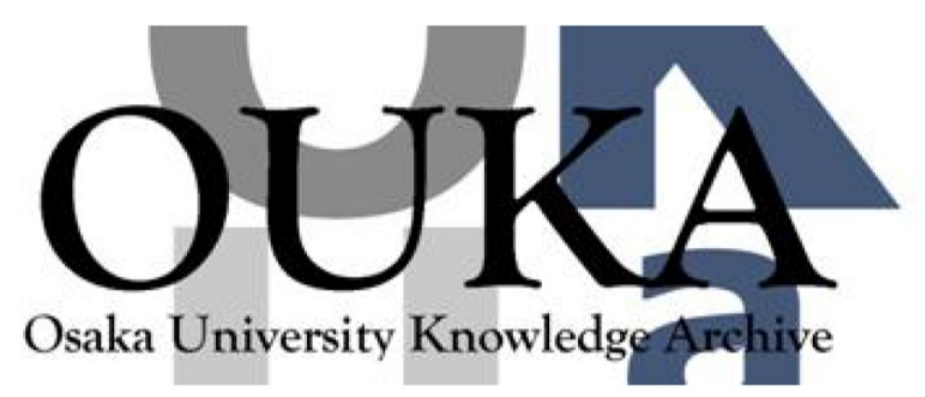

\begin{tabular}{|c|c|}
\hline Title & $\begin{array}{l}\text { Alpha-Transfer Reaction with Large Energy } \\
\text { Transfers }\end{array}$ \\
\hline Author(s) & Fröhlich, H.; Shimoda, T.; Ishihara, M. et al. \\
\hline Citation & Physical Review Letters. 42(23) p. 1518-p. 1520 \\
\hline Issue Date & $1979-06-04$ \\
\hline oaire:version & VoR \\
\hline URL & https://hdl. handle. net/11094/23124 \\
\hline rights & $\begin{array}{l}\text { Fröhlich, H. , Shimoda, T. , Ishihara, M. , } \\
\text { Nagatani, K. , Udagawa, T. ', Tamura, T..' } \\
\text { Physical'Review Letters, 42, 1518-1520, } 1979 \\
\text { "Copyright (1979) by the American Physical } \\
\text { Society." }\end{array}$ \\
\hline Note & \\
\hline
\end{tabular}

Osaka University Knowledge Archive : OUKA

https://ir. Library. osaka-u. ac. jp/

Osaka University 


\title{
Alpha-Transfer Reactions with Large Energy Transfers
}

\author{
H. Fröhlich, (a) T. Shimoda, (b) M. Ishihara, ${ }^{(c)}$ and K. Nagatani \\ Cyclotron Institute, Texas A\&M University, College Station, Texas 77843 \\ and \\ T. Udagawa and T. Tamura \\ Department of Physics, University of Texas, Austin, Texas 78712
}

(Received 1 February 1979)

\begin{abstract}
Alpha-transfer reactions $\left({ }^{20} \mathrm{Ne},{ }^{16} \mathrm{O}\right),\left({ }^{14} \mathrm{~N},{ }^{10} \mathrm{~B}\right)$, and $\left({ }^{13} \mathrm{C},{ }^{9} \mathrm{Be}\right)$ on a ${ }^{40} \mathrm{Ca}$ target were studied at 262,153 , and $149 \mathrm{MeV}$, respectively. Analysis in terms of the direction-reaction theory reproduced the observed continuum spectra and angular distributions well, except for the cross section of the reaction $\left({ }^{20} \mathrm{Ne},{ }^{16} \mathrm{O}\right)$ at small angles, which is attributed to a projectile breakup process.
\end{abstract}

Heavy-ion reactions with bombarding energies well above the Coulomb barrier generally produce continuum spectra. One of the intriguing features in such reactions is the appearance of enhanced cross sections at ejectile energies considerably lower than the incident energy; that is, the process involves a large energy transfer. The surprising appearance of the deeply inelastic process has lead to various theoretical attempts introducing a variety of concepts, such as frictional forces, mass and energy transport, and so forth. ${ }^{1}$

Recently, however, a quantum mechanical treatment using the direct-reaction model, which has been routinely applied to analyses for discrete spectra, was extended to analyze data with continuum spectra. ${ }^{2-4}$ If this method can demonstrate a wide range of applicability, especially for reactions of a deeply inelastic nature, it will provide a new theoretical approach for understanding the heavy-ion reaction. It should also be pointed out that the application of the direct-reaction theory, which is coupled to a microscopic description of the nuclear structure, may open up the possibility of using the heavy-ion reaction in the study of nuclear structure in the continuum region, such as the extraction of the spectroscopic density of multinucleon states with very high spins in high excitation regions of the residual nuclei. The choice of the present $\alpha$-transfer reactions was made for specific reasons.

The projectiles ${ }^{20} \mathrm{Ne},{ }^{14} \mathrm{~N}$, and ${ }^{13} \mathrm{C}$ are known to have large $\alpha$-transfer spectroscopic amplitudes. In addition, the angular momentum $\left(L_{\alpha}\right)$ of the cluster relative to the core in ${ }^{20} \mathrm{Ne},{ }^{13} \mathrm{C}$, and ${ }^{14} \mathrm{~N}$ is dominantly 0,2 , and 4 , respectively. As discussed later, the difference in the $L_{\alpha}$ values results in a subtle difference in the spectral shape. Therefore, a simultaneous study of the $\alpha$-transfer reactions with these projectiles on the same target should impose a stringent test on the theoretical analysis.

The experiments were carried out using 262$\mathrm{MeV}{ }^{20} \mathrm{Ne}^{6+}, 153-\mathrm{MeV}^{14} \mathrm{~N}^{4+}$, and $149-\mathrm{MeV}^{13} \mathrm{C}^{4+}$ beams from the Texas A\&M cyclotron on a selfsupporting natural calcium target of $1.0-\mathrm{mg} / \mathrm{cm}^{2}$ thickness. Outgoing particles were detected using solid-state counter telescopes. Some of the energy spectra are shown in Fig. 1, and the angular distributions of the energy-integrated cross sections are displayed in Fig. 2. Several observations can be made from these spectra. Each shows a continuum spectrum and, within the statistics, no discrete peaks are observed. In the reactions $\left({ }^{14} \mathrm{~N},{ }^{10} \mathrm{~B}\right)$ and $\left({ }^{13} \mathrm{C},{ }^{9} \mathrm{Be}\right)$, the angular dependence of the spectral shapes is weak. On the other hand, at small angles the spectra of the reaction $\left({ }^{20} \mathrm{Ne},{ }^{16} \mathrm{O}\right)$ show an extra hump in the highenergy region; this disappears at large angles. This difference is more conspicuous in the angular distribution of the reaction $\left({ }^{20} \mathrm{Ne},{ }^{16} \mathrm{O}\right)$ which has a steeper slope in forward angles as compared with the other two reactions. Remarkably, the magnitude of the forward-angle cross section of the reaction $\left({ }^{20} \mathrm{Ne},{ }^{16} \mathrm{O}\right)$ is much larger than those of the other reactions.

Theoretical cross sections were calculated by using an exact-finite-range (EFR) distorted-wave Born-approximation (DWBA) method, assuming direct $\alpha$-transfer processes leading to many overlapping continuum states. The details of the method have already been explained ${ }^{2-4}$; several salient points are noted below. The continuum cross section is given as a sum of products of the EFRDWBA cross sections and the $\alpha$-spectroscopic densities in the same residual nuclei of ${ }^{44} \mathrm{Ti}$, where the sum is taken over the spins of the final states. In the EFR-DWBA calculation, the overlap integrals are expressed in an analytic form, which includes the quantum numbers and the $Q$ 


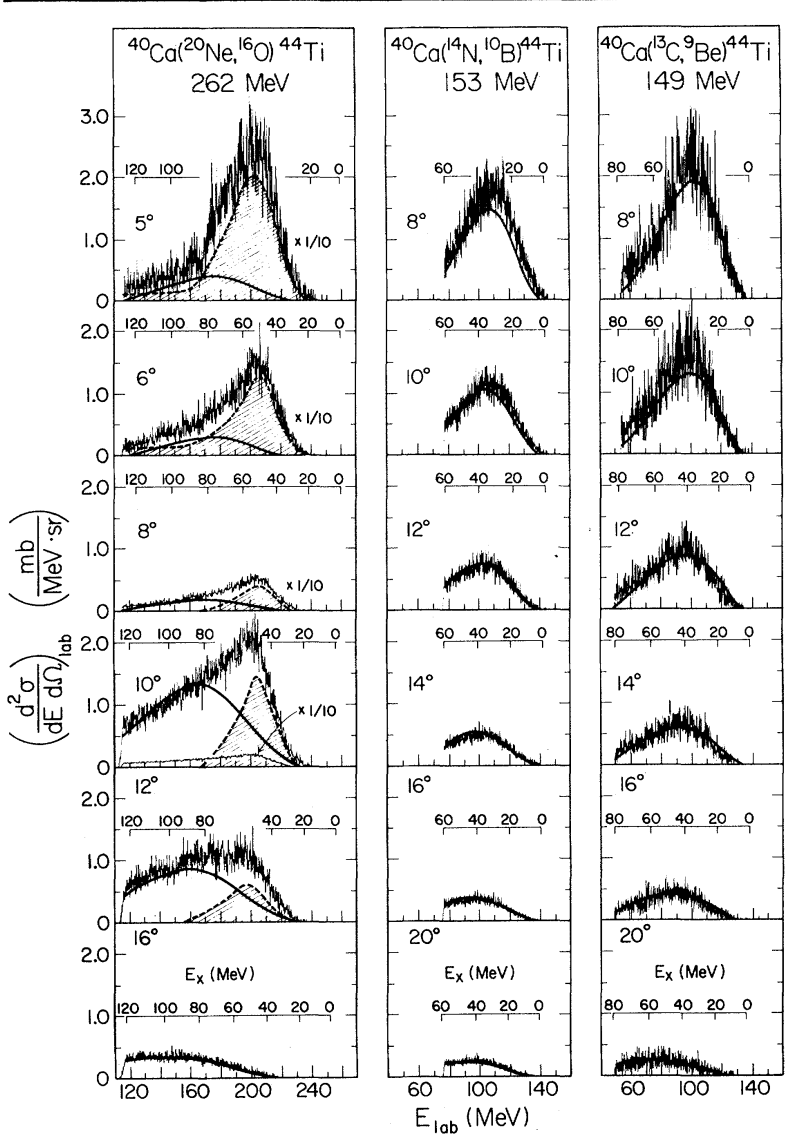

FIG. 1. Energy spectra in the laboratory systems. Each spectrum is labeled by the laboratory angle, and the excitation energy in the residual ${ }^{44} \mathrm{Ti}$ system is indicated by the $E_{x}$ scale inserted. The solid lines are the calculated spectra normalized at one angle for each reaction. The dashed lines are hatched ares in the reaction $\left({ }^{20} \mathrm{Ne},{ }^{16} \mathrm{O}\right)$ demonstrate the differences between the experimental spectra and the calculated spectra which are normalized at the backward angles.

values, as well as several parameters..$^{2-4}$ EFRDWBA calculations were made for a few thousand sets of quantum numbers and $Q$ values; this was followed by a $\chi^{2}$ fitting procedure to determine the parameters. The cross-section calculations could then be carried out with considerable speed (it typically takes one minute on CDC-6600 computer to obtain a spectrum), while maintaining sufficient accuracy. Note that the use of these parametrized overlap integrals replaces the ordinary numerical integrals for several million sets of the quantum numbers. As to the spectroscopic density, several models were investigated, and it was found that the density derived from the SU(3) shell model ${ }^{5}$ best reproduced the observed spectra. Therefore, the density used throughout

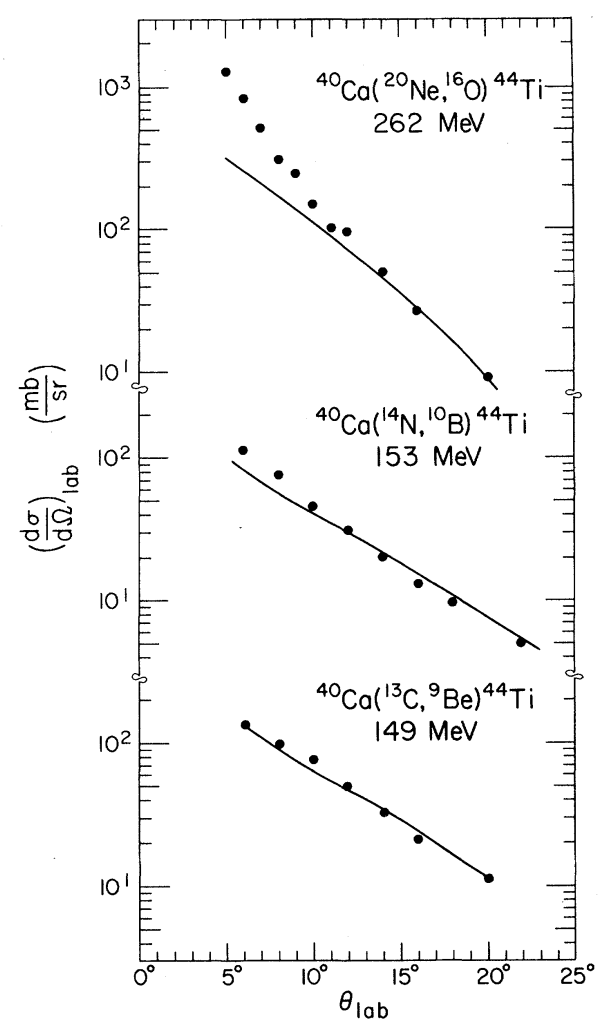

FIG. 2. Angular distributions in the laboratory systems. The energy-integrated cross sections down to the cutoff energies are shown by the solid circles, while the calculated results are shown by the solid lines. The normalizations are made the same as in Fig. 1.

the entire course of the calculation was fixed in this form, that it, it was common for all the reactions studied.

The solid lines in Fig. 1 represent the theoretical cross sections thus obtained. An overall normalization constant was introduced separately for each reaction such that for the reactions $\left({ }^{14} \mathrm{~N},{ }^{10} \mathrm{~B}\right)$ and $\left({ }^{13} \mathrm{C},{ }^{9} \mathrm{Be}\right)$ overall fits were obtained in the entire angle range. For the reaction $\left({ }^{20} \mathrm{Ne},{ }^{16} \mathrm{O}\right)$ the normalization was chosen to fit the large-angle $\left(\gtrless 14^{\circ}\right)$ spectra, by which the cross section at forward angles is underestimated. The dashed curves and hatched areas display the difference between the theoretical and experimental cross sections. It was found that those normalization factors agree each other within a factor of 10 , which certainly warrants the validity of the present calculations considering the difficulty and uncertainty involved in evaluating the magnitudes of cross sections. In Fig. 2 comparisons are made between the experimental and theoretical distribu- 
tions of energy-integrated cross sections. As obviously expected, from Fig. 1, the angular distributions are well fitted except for the reaction $\left({ }^{20} \mathrm{Ne},{ }^{16} \mathrm{O}\right)$ at forward angles.

Since this discrepancy in the ${ }^{20} \mathrm{Ne}$-induced reaction exists only in the forward angles and rapidly disappears at backward angles, it may be reasonably conjectured that the origin of this anomalous part of the cross section corresponds to the direct breakup of the projectile. The fact that the ${ }^{20} \mathrm{Ne}$ nucleus is one of the most $\alpha$-like nuclei seems to enhance the argument. It should also be noted that in the present theoretical treatment the breakup process was excluded because of the use of a form factor corresponding to the $\alpha$ particle confined to the surface region, as previously explored in Refs. 2-4. By relaxing this restriction, the theory can be extended to include the contributions of such a breakup process, and a study of this is now under way.

It was mentioned above that these reactions are initiated from different cluster modes in the projectiles with $L_{\alpha}=0,2$, and 4 , respectively, for ${ }^{20} \mathrm{Ne},{ }^{13} \mathrm{C}$, and ${ }^{14} \mathrm{~N}$. Detailed examination of the analysis showed that the transition strength strongly depends on these $L_{\alpha}$ values especially their magnetic substates-as is reflected by a subtle difference in the shapes of the spectra of these reactions: For instance, had we chosen $L_{\alpha}=2$ for ${ }^{14} \mathrm{~N}$, we would have obtained poorer fits to the data for the spectra of the reaction $\left({ }^{14} \mathrm{~N}\right.$, $\left.{ }^{10} \mathrm{~B}\right)$.

In conclusion, despite the large energy losses involved, the continuum spectra presented here can be successfully interpreted as resulting from direct-transfer reactions. Note that the elastic scattering of the present collisions have their grazing angles at about $5^{\circ}$ to $8^{\circ}$; thus all the ob- served spectra were taken much beyond the grazing angles. If a macroscopic model, such as that of Wilczynski, ${ }^{6}$ had been used, an entirely different feature would have been predicted for the present reactions; the present approach, in terms of the direct-reaction theory, explains the experimental spectral shapes and angular distributions and provides a quantitative understanding. The discrepancy seen in the reaction $\left({ }^{20} \mathrm{Ne},{ }^{16} \mathrm{O}\right)$ analysis is not considered to be a drawback of the method. In fact, the possibility of extending the analysis to include the breakup process is an advantage which demonstrates the flexibility built into the method.

The present work was supported in part by the National Science Foundation and the U. S. Department of Energy.

\footnotetext{
(a) Permanent address: Physikalisches Institut, Universität Erlangen-Nürnberg, Erlangen, West Germany.

(b) Permanent address: Department of Physics, Kyoto University, Kyoto, Japan.

(c) Permanent address: Institute of Physical and Chemical Research, Wakoshi, Saitama, Japan.

${ }^{1}$ See review articles by W. U. Schröder and J. R. Huizenga, Ann. Rev. Nucl. Sci. 27, 465 (1977); N. Lefort and Ch. Ngo, Ann. Phys. (Paris) 3 , 5 (1978).

${ }^{2}$ T. Udagawa, B. T. Kim, and T. Tamura, in Proceedings of the Institute of Physical and Chemical Research Symposium, Hakone, Japan, September 1979, edited by H. Kamitsubo and M. Ishihara (unpublished), p. 3.

${ }^{3}$ T. Udagawa, T. Tamura, and B. T. Kim, to be published.

${ }^{4} \mathrm{~T}$. Udagawa and T. Tamura, Phys. Rev. Lett. $\underline{41}$, 1770 (1978), and references therein.

${ }^{5}$ M. Ichimura, A. Arima, E. C. Halbert, and T. Terasawa, Nucl. Phys. A204, 225 (1973); T. Tamura and

T. Udagawa, Phys. Lett. 71B, 273 (1977).

${ }^{6}$ J. Wilczyński, Phys. Lett. 47B , 484 (1973).
}

\title{
Proposal for an Intense Polarized Li-Ion Source
}

\author{
L. W. Anderson and George A. Nimmo \\ Department of Physics, University of Wisconsisn, Madison, Wiscon 53706 \\ (Received 12 March 1979)
}

\begin{abstract}
We propose to use optical pumping with a high-power dye laser to produce the polarized $\mathrm{Li}$ atoms necessary for a polarized $\mathrm{Li}$ ion source. It should be possible to produce very intense beams of highly polarized Li ions.
\end{abstract}

There are a variety of polarized-ion sources being used for nuclear-reaction experiments at the present time. ${ }^{1}$ One of these is a polarized
${ }^{6} \mathrm{Li}^{-}$ion source that was developed at Heidelberg. ${ }^{2}$ In that source thermal ${ }^{6} \mathrm{Li}$ atoms emerge from an oven through a Laval nozzle and after being polar- 